\section{nephron \\ Clinical}

Practice
Nephron 2019;143:193-196

DOI: $10.1159 / 000500550$
Received: March 15, 2019

Accepted after revision: April 24, 2019

Published online: May 16, 2019

\title{
Acute Kidney Injury in Kidney Transplants: New Insights
}

\author{
Roslyn B. Mannon \\ Division of Nephrology, University of Alabama at Birmingham, Birmingham, AL, USA
}

\section{Keywords}

Transplant · Acute kidney injury · Outcomes · Creatinine · Allograft · Failure

\section{Abstract}

Delayed allograft function (DGF) is defined as dialysis treatment in the kidney transplant recipient in the first week following transplantation. With the demand for kidney transplants growing and the supply limited, as well as implementation of a national allocation scheme for deceased donor kidneys, rates of DGF remain high, on average, 30\% for recipients of deceased donor kidneys. DGF is associated with inferior allograft outcomes, and there are no FDA-approved therapies to mitigate this disorder. There is renewed interest in this therapeutic arena, and there are several recent clinical trials that have considered interventions within the recipient to reduce injury. A critical issue is that of trial design and end points as well as translating from acute kidney injury (AKI) trials in cardiac bypass to the more complicated kidney transplant scenario. DGF is a significant clinical outcome after kidney transplantation without known approved therapy beyond clinical support. This mini-review highlights our presentation at the 24th International Conference on Advances in Critical Care Nephrology and UAB/UCSD O'Brien Center AKI Pre-Meeting.

(c) 2019 S. Karger AG, Basel

\section{Introduction}

Transplantation of a deceased donor kidney is associated with a significantly higher risk of acute kidney injury (AKI) following implantation and reperfusion. This risk is attributed to a number of features found in both donor and recipient including both cold and warm ischemia [1] and interplay of both innate and adaptive immunity [2].

Contribution from the AKI and CRRT 2019 Symposium at the 24th International Conference on Advances in Critical Care Nephrology, Manchester Grand Hyatt, San Diego, CA, USA, February 26 - March 1 , 2019. This symposium was supported in part by the NIDDK-funded University of Alabama at Birmingham-University of California San Diego O'Brien Center for Acute Kidney Injury Research (P30DK079337).

\section{KARGER}

(C) 2019 S. Karger AG, Basel

E-Mail karger@karger.com

www.karger.com/nef
Roslyn B. Mannon, MD Division of Nephrology University of Alabama at Birmingham, 1900 University Boulevard THT 643, Birmingham, AL 35294 (USA)

E-Mail rmannon@uabmc.edu 
Dialysis treatment of the kidney transplant recipient (KTR) in the first 7 days of transplantation is termed "delayed allograft function" (DGF). DGF is associated with higher rates of allograft loss and rejection, although the risk appears dependent on the frequency of dialysis treatments and persistence of organ failure [3]. In spite of much investigation, there are still no regulatory-approved therapies to mitigate injury. Opportunities include donor intervention and management, recipient intervention and management, as well as manipulation and treatment of the organ, ex vivo, following recovery. The extensive ethical and regulatory barriers in donor management research are highlighted elsewhere [4] and remain a novel and exciting avenue for future inroads to improve organ function in the brain-dead donor. Ex vivo therapies may prove to be the most important in terms of salvaging organs before a point of no return [5]. The focus of this mini-review will be on strategies in the KTR to limit AKI and improve kidney function after transplantation.

\section{Approach to the Transplant Recipient: Clinical Stabilization}

Historically, the KTR has been the focus of management in the perioperative period to minimize hemodynamic dysfunction that worsens ischemic injury. Strategies have focused on volume expansion with variable and debated strategies, and there are no specific consensus guidelines in this regard. Pretransplant dialysis for electrolyte correction tends to avoid excessive volume removal. Another strategy is pressor support. While "renal dose" dopamine has been clinically implemented and no harm detected, it has not been found to be effective in terms of minimizing DGF [6]. The final objective in recipient management is the choice of immunosuppression. As calcineurin inhibitors (CNIs) may augment renal ischemia through vasoconstriction, clinicians avoid implementation when the concern for DGF is present to minimize prolongation of renal injury. It has been common practice in North America to utilize lymphocyte depletional induction with rabbit antithymocyte globulin (rATG) or campath, with the notion that such treatments would delay implementation of CNIs. Further, ATG might potentially mitigate innate and adaptive immune molecules through depletion of mononuclear cells and blockade of costimulatory ligands. This has not proven the case based on randomized trials, even with meta-analysis [7]. Indeed, a randomized clinical trial known as "PREDICT" (NCT02056938) was designed to compare
rATG induction to nondepletional induction with basiliximab and delayed CNI initiation in patients at high risk for DGF, but the trial was terminated due to poor enrollment. Still, the vast majority of transplant centers in North America utilize rATG in this setting with delayed CNI initiation.

\section{Injury and Inflammation: The Focus of Direct Clinical Therapies}

Innate immune activation pathways are critical to mediating injury [2], associated with complement activation, adhesion molecule expression on the organ endothelium, release of pathogen-associated molecular patterns, and a pro-coagulant microenvironment. These pathways cross talk to the adaptive immune response leading to cellular rejection. Global therapies studied have included the use of inhaled carbon monoxide, disruption of cell adhesion molecule to minimize immune cell adhesion and sequestration in injured tissues using a recombinant $\mathrm{P}$ and $\mathrm{E}$ selectin ligand, or induction therapy with anti-intracellular adhesion molecule 1 antibody. None of these therapies translated effectively into further clinical studies in man in spite of strong preclinical rodent data suggesting impacts in both immune-mediated injuries.

More encouraging data exist with complement inhibitors. These agents have a remarkable pipeline and have an impact on both innate and alloimmune responses (reviewed in [8]). Preclinical data highlight complement activation in the setting of ischemia reperfusion injury, and human studies identify complement upregulation with quantitative differences between living and deceased brain-dead donors [9]. While still early phase trial, Jordan et al. [10] demonstrated that treatment with C1-esterase inhibitor at the time of transplant and $24 \mathrm{~h}$ later in 70 randomized patients at risk for DGF did not reduce DGF incidence but reduced the number and duration of dialysis treatments without associated safety concerns with significantly improved renal function 1 year later. An alternative strategy that is downstream in the complement cascade has been the use of anti-C5 antibody (eculizumab) to prevent DGF. In a randomized, double-blind controlled study ("PROTECT" trial; NCT02145182), 286 KTRs received eculizumab or placebo prior to reperfusion and posttransplantation. The incidence of DGF, death, graft loss, or loss to follow-up at 7 days posttransplant was $35.9 \%$ on treatment, compared to $41.7 \%$ for patients receiving placebo $(p=0.398)$. However, serious ad- 
verse events in the first 60 days of treatment were lower for patients in the eculizumab group (44.4\%) compared to control (54.1\%; submitted for review). This disappointing result may relate again to the study design, wherein 400 randomized subjects were initially aimed for, but recruitment fell well short. It appears unlikely that further trials will be considered at this time, and further post hoc analysis is pending.

Finally, promising preclinical studies of hepatocyte growth factor in ameliorating AKI [11] have led to the initiation of an ongoing phase 3 clinical trial in KTRs (NCT02474667). Treatment with the investigative agent BB3 initiated posttransplant in those with poor allograft function and limited urine output in a randomized, double-blind, placebo-control fashion. Targeted cases with be those KTRs with "poor" renal function in the first $24 \mathrm{~h}$ posttransplantation based on an average urine output $<50 \mathrm{~mL} / \mathrm{h}$ over any 8 consecutive hours, to maximize the likelihood that the patient requires dialysis within the first 7 days posttransplant.

\section{Cell Death in Mediating AKI}

Elegant work by a number of laboratories has highlighted the complexity of cell death pathways in mediating renal injury following ischemia/reperfusion [12] and the immunological reactivity of regulated necrosis (necroptosis) [13]. While each of these topics is individually worthy of a separate detailed discussion, it is apparent that encouraging preclinical studies may not translate easily into humans and that multiple pathways will need to be targeted to extract a clinical benefit. This has been reflected by the relative lack of success in human randomized trials of DGF, for example, using diannexin, a biosynthesized homodimer of human annexin V, binds phosphatidylserine that is translocated to the cell surface during ATPdepletion/injuryofapoptosis(NCT01442337).

More recently, there is interest in a silencing messenger RNA to tumor suppressor p53 (QP-1002), a key partner in both intrinsic and extrinsic pathways of apoptosis. A phase 1 study in 40 KTRs demonstrated no dose-limiting toxicity, and a phase 2 study in 32 KTRs was encouraging. Additive were the findings of a phase 2 study of 341 patients undergoing cardiac bypass with a striking and significant $26 \%$ reduction in AKI in treated group (presented at 2017 Kidney Week of the American Society of Nephrology; Abstract SA-OR124). However, a recently completed phase 3 randomized, double-blind, placebo-controlled study of 634 KRTs to evaluate the efficacy and safety of QPI-1002 for the prevention of DGF ("ReGIFT"; NCT02610296) was disappointing in not achieving the primary end point to reduce frequency of DGF (personal communication). These studies highlight not only the complexity of clinical translation but also the potential differences in trial execution in transplantation compared to a known ischemic insult.

\section{The Failure of Clinical Trials in DGF}

Despite extensive experimental investigation, no therapy is approved for use in KTRs to reduce DGF. Translational disconnect exists between the preclinical models used and the typical clinical problems encountered in transplant recipients. Clinical trials conducted to date have included small numbers of renal transplant recipients, heterogeneous early end points, and tested single agents in isolation.

Scientific knowledge of IRI is rapidly evolving and new treatments are emerging. The Food and Drug Administration recently published guidance to industry in developing therapeutics to reduce DGF [14]. Key points of the report include creating superiority trials in which active treatment is compared with placebo using a study population that is representative of a US patient population, including race, age, sex, and other baseline characteristics, and donors graded based on the United Network of Organ Sharing Kidney Allocation scheme. Recommendations include stratifying study enrollment based on (1) study center, type of induction treatment (if more than 1), (2) organ type and organ preservation method (highly recommended), and (3) enrichment strategies can be used to select the study population at higher risk for developing DGF compared to overall KTR population. Considerations were also provided for short-term assessment or composite end point that include the common definition of DGF (i.e., hemodialysis within week 1 posttransplantation) or other definitions supported by literature such as a DGF severity scoring system including number of hemodialysis sessions required until recovery of renal function and time to recovery of renal function after diagnosis of DGF. Other potential short-term composite end points may include slow graft function and functional DGF with protocol-specified definitions and clinically meaningful justification.

The complexity of brain death and resultant DGF has further complicated study designs and recruitment. With the plethora of preclinical studies identifying novel 
mechanisms amenable to treatment, we must remain cognizant of the unmet need to improve DGF and the challenges in such studies so that innovation and improved organ utilization can accelerate in the coming decade.

\section{Disclosure Statement}

The author has received research grant support from Alexion and Quark Pharmaceuticals.

\section{References}

1 Mannon RB. Delayed Graft Function: The AKI of Kidney Transplantation. Nephron. 2018;140(2):94-8.

2 Siedlecki A, Irish W, Brennan DC. Delayed graft function in the kidney transplant. Am J Transplant. 2011 Nov;11(11):2279-96.

3 Gill J, Dong J, Rose C, Gill JS. The risk of allograft failure and the survival benefit of kidney transplantation are complicated by delayed graft function. Kidney Int. 2016 Jun; 89(6):1331-6.

4 National Academies of Sciences, Engineering, and Medicine; Health and Medicine Division; Board on Health Sciences Policy; Committee on Issues in Organ Donor Intervention Research. Medicine. Opportunities for Organ Donor Intervention Research. In: James FC, Sarah D, Catharyn TL, editors. Saving Lives by Improving the Quality and Quantity of Organs for Transplantation. Washington (DC): The National Academies Press; 2017.

5 Kaths JM, Paul A, Robinson LA, Selzner M. Ex vivo machine perfusion for renal graft preservation. Transplant Rev (Orlando). 2018 Jan;32(1):1-9.

6 Schnuelle P, Benck U, Yard BA. Dopamine in transplantation: written off or comeback with novel indication? Clin Transplant. 2018 Jul; 32(7):e13292.

7 Liu Y, Zhou P, Han M, Xue CB, Hu XP, Li C. Basiliximab or antithymocyte globulin for induction therapy in kidney transplantation: a meta-analysis. Transplant Proc. 2010 Jun; 42(5):1667-70.

8 Ricklin D, Mastellos DC, Reis ES, Lambris JD. The renaissance of complement therapeutics. Nat Rev Nephrol. 2018 Jan;14(1):2647.

9 Naesens M, Li L, Ying L, Sansanwal P, Sigdel TK, Hsieh SC, et al. Expression of complement components differs between kidney allografts from living and deceased donors. J Am Soc Nephrol. 2009 Aug;20(8):183951.

10 Jordan SC, Choi J, Aubert O, Haas M, Loupy A, Huang E, et al. A phase I/II, double- blind, placebo-controlled study assessing safety and efficacy of $\mathrm{C} 1$ esterase inhibitor for prevention of delayed graft function in deceased donor kidney transplant recipients. Am J Transplant. 2018 Dec;18(12): 2955-64.

11 Narayan P, Duan B, Jiang K, Li J, Paka L, Yamin MA, et al. Late intervention with the small molecule BB3 mitigates postischemic kidney injury. Am J Physiol Renal Physiol. 2016 Aug;311(2):F352-61.

12 Hébert MJ, Jevnikar AM. The Impact of Regulated Cell Death Pathways on Alloimmune Responses and Graft Injury. Curr Transplant Rep. 2015;2(3):242-58.

13 Linkermann A, Chen G, Dong G, Kunzendorf U, Krautwald S, Dong Z. Regulated cell death in AKI. J Am Soc Nephrol. 2014 Dec;25(12): 2689-701.

14 Food and Drug Administration. Delayed Graft Function in Kidney Transplantation: Developing Drugs for Prevention Guidance for Industry. Silver Spring, MD; 2017. 